\title{
A Test System for Hidden Failure Based on Hybrid State Estimation
}

\author{
Zhongqing $\mathrm{Li}^{1, \text { a }}$, Yuan Tian ${ }^{2, b^{*}}$, Chen Liang ${ }^{3, \mathrm{c}}$, Yan $\mathrm{Hu}^{4, \mathrm{~d}}$, Xiang Gao,e \\ ${ }^{1}$ China Electric Power Research Institute, Beijing, 100192 \\ ${ }^{2}$ School of Electronic, Information and Electrical Engineering, Shanghai Jiao Tong University, \\ Shanghai 200240 \\ ${ }^{3}$ Shanghai No.1 Automation Company, Shanghai, 201204), \\ azqing@epri.sgcc.com.cn, b ryomasusan@163.com, ${ }^{\mathrm{c}} 494459210 @ q q . c o m$
}

\begin{abstract}
Keywords: power system management; hidden failure; state estimation; static condition; WAMS Abstract. Hidden failures in relay may cause invalidation of protection equipment so that system faults cannot be detected. These faults will lead to cascading outages in severe cases. At present, effective methods lack in monitoring hidden failures. A hybrid state estimation which uses system data collected from both SCADA and WAMS is proposed. The state value obtained from state estimation is regarded as reference value. The measurement value uploaded by protection information system is compared with reference value. If the difference exceeds default threshold value, hidden failure can be proved to exist in relay. A test system for hidden failure is established according to analysis above. This system can be used on the long-term, real-term, and on-line monitoring of relay. Example demonstrates that this system can be used to check the hidden failure in relay effectively.
\end{abstract}

\section{Introduction}

Hidden failures in relay will lead to mal-operation or refused operation of protection equipment ${ }^{[1-3]}$. Sometimes these serious faults will even lead to cascading outages. In order to improve the operation reliability of power grids, it is necessary to monitor the possible hidden failures in protection equipment. So far, the articles published at home and abroad have put forward ideas about monitoring and preventing hidden failures. Due to the restriction on technical development, however, further study has not been carried out ${ }^{[4-9]}$. The paper5, 6 mainly studies the probability of hidden failures, and a cascading failure model considering hidden failures has been built. The paper7, 8 sets up a method to analyze and prevent cascading failures in power system based on searching model and fault tree. But none of above puts forward the specific method to detect and prevent hidden failures in protection equipment.

With the development of communication technology, high-precision measurement units are widely applied in power system ${ }^{[10,11]}$. The wide area measurement system(WAMS) which uses GPS-based phase measurement units(PMU) to acquire synchronous operation states of power system can obtain the states in real time. Besides, domestic and foreign experts already have intense researches on PMU optimization placement and data analysis, which could guarantee the observability of power grid ${ }^{[12]}$. The power system data obtained by WAMS can be used as ideal data sources to analysis the states of power grid. And there are advantages of high sampling rate and high precision. What's more, the SCADA system based on RTU can monitor the operation state of power system along with WAMS. The protection information system is used to collect, analyze and process information of protection equipment ${ }^{[14,15]}$, as well as record measurement data and trigger 
signals at the same time to monitor the operation status of protection equipment. The above systems can be used to check the hidden failure in protection equipment comprehensively.

Starting from the operational principle of protection equipment, reference 9 points out that the operation states of devices can be divided into static states before power grid failure and dynamic states after power grid failure. These two states put up static characteristic and dynamic characteristic. The paper also presents a method which compares the power system data measured by WAMS and the data obtained from protection information system to detect the hidden failures in protection equipments under static condition. However, this method can only be used for detecting the hidden failures of single bus, and cannot guarantee the reliability of data. And exceptions in WAMS may lead to unreliable data which will cause misjudgment of hidden failure. Therefore, this paper puts forward a method to detect hidden failure in protection equipment under static condition. It uses hybrid state estimation to process data from both WAMS and SCADA so as to conclude the operation states of power grid. Compare these state data with protection data recorded by protection information system. If there is an obvious difference, the protection equipment may have hidden failures. Then a system used to detect hidden failure in protection equipment is established on the basis of it. The system can constantly detect the hidden failures under normal operation of power network on line, and effectively reduce the influence of abnormal power measurement data on detection. It is of good practical value.

\section{The test system for hidden failure}

Hidden failure can be proved to exist in different segments of protection equipment. The methods put forward to detect hidden failure should consider the differences between each part. When the power system operates steadily, the protection equipment cannot reach the starting conditions. The protection program may operate under normal process. At this time, the protection equipment runs under static condition. Its main function is to measure current and voltage. The hidden failures mainly manifest that the analog data measured by protection and actual measured data are different, but the protection equipment by no means detect the abnormalities. If the hidden failure can be detected to ensure the correct static characteristic, the faulty operation and missing operation of protection equipment can be avoided. Checking the accuracy of measurement data is a better way to find hidden failure.

This kind of defects will make deviation when power system operates in normal condition. When load current increases immediately or large disturbance occurs in grid, the existence of bias might make the protection equipment sending a wrong tripping signal. Consequently, when protection equipment operates under static condition, the detecting method for hidden failure is mainly to detect the defects in data acquisition and processing.

\section{Hybrid State Estimation}

It needs reliable contrast resource to monitor the data collected and processed by relay protection equipment. The paper 16 set up a method which could obtain reliable operation state of system by using data collected from SCADA and WAMS to estimate the state of system. The advantage of state estimation is that bad data can be detected and identified through redundant data. The state variable in this method has high precision. It can be used as the comparing data resource to the detecting system. Comparing the measurement value of protection equipment with the reference value, if the result exceeds default threshold value, hidden failure can be proved to exist in the measurement part of protection equipment. 
By using system data from SCADA to solve nonlinear equation group, the optimum estimated states of system can be obtained. When the measurement error is subject to uncorrelated normally distribution, the mean is 0 , and the variance is $\sigma^{2}$, and the measurement equation of SCADA system is

$$
z=h(U, \theta)+v
$$

In this equation, $\mathrm{z}$ is m-dimensional measurement, and usually is the power injected to nodes or power flow. The bus voltage amplitude $\mathrm{U}$ and Phase Angle $\theta$ are n-dimensional measurement. $\mathrm{V}$ is measurement error. So the state estimation goal function is:

$$
J(U, \theta)=\sum_{i=1}^{m}\left(\frac{z_{i}-h_{i}(U, \theta)}{\sigma_{i}}\right)^{2}=[z-h(U, \theta)]^{\mathrm{T}} R^{-1}[z-h(U, \theta)]
$$

In this equation, $R^{-1}$ is weight matrix. This matrix is diagonal, and its diagonal elements are $1 / \sigma_{i}^{2}$.

For power measurement, the correction equation of measurement is:

$$
\left[\begin{array}{c}
\Delta P \\
\Delta Q
\end{array}\right]=H\left[\begin{array}{c}
\Delta U / U \\
\Delta \theta
\end{array}\right]=\left[\begin{array}{cc}
M & F \\
G & N
\end{array}\right]\left[\begin{array}{c}
\Delta U / U \\
\Delta \theta
\end{array}\right]
$$

In this equation, $\mathrm{M}, \mathrm{F}, \mathrm{G}, \mathrm{N}$ are all the first order partial derivative matrix of the power injected to nodes or power flow with respect to the voltage amplitude and phase angles. Each row has two nonzero elements. The nonzero element of nodes $i$ or $j$ is located in the column $i$ or column $j$. With regard to the nodes configured by PMU, the additional correction of branch power flow can be constructed to improve the redundancy and the precision of the data ${ }^{[16 \sim 18]}$. After introducing the PMU measurement data, the correction equation can be rewritten:

$$
\left[\begin{array}{c}
\Delta P \\
\Delta Q \\
\Delta P_{a} \\
\Delta Q_{a}
\end{array}\right]=\left[\begin{array}{cc}
M & F \\
G & N \\
M_{a} & F_{a} \\
G_{a} & N_{a}
\end{array}\right]\left[\begin{array}{c}
\Delta U / U \\
\Delta \theta
\end{array}\right]
$$

If one node $\mathrm{i}$ or $\mathrm{j}$ at each end is equipped with PMU, only one of the nonzero elements of the row vector of $\mathrm{Ma}, \mathrm{Fa}, \mathrm{Ga}, \mathrm{Na}$ is located in the i column or $\mathrm{j}$ column. If both nodes $\mathrm{i}, \mathrm{j}$ are equipped with PMU, Ma, Fa, Ga, Na have the same structure as M、F、G、N. Introducing the PWU could improve the redundancy of data. And high precision of PMU measurement could improve the precision and stability of state estimation. If the precision of PMU measurement is high enough, the measurement data can be directly used as the state solution of the system. In the meantime, the number of state variables in state estimation will reduce. The computational amount is reduced. And the precision is further improved. In point of the whole power grid, state estimation could reduce the test error of each bus so as to improve the precision of referential values of hidden failures.

The results of state estimation are the measurement estimates $\hat{U}, \hat{\theta}$, the phase angle and magnitude of each bus voltage. Thus, the measurement estimates of voltage phasor $\hat{U} \angle \theta$ of each bus can be obtained. Then the measurement estimate of current vector of every branch is:

$$
\hat{I}_{i j} \angle \theta_{i j}=Y_{i j}\left(\hat{U}_{i} \angle \theta_{i}-\hat{U}_{j} \angle \theta_{j}\right)
$$


In this equation, $Y_{i j}$ is the conductance between buses $\mathrm{i}$ and $\mathrm{j} . \theta_{i j}$ is the degrees that the branch current lags behind the reference voltage.

The normal measurement data that protection equipments upload onto the protection information system are the amplitudes of voltage and current. According to the analysis above, the calculated value of state estimation can be obtained. And they will be used as referential values to detect hidden failures in protection equipment under static condition.

\section{Detecting Hidden Failure under Static Condition}

Power system is an inertia system which contains a large number of generators. And its steady operation changes slowly. The hidden failures are detected when the power system operates steadily. There has the observability of power grid in WAMS and SCADA system. The measurement data such as bus voltage and current detected from power grid should keep stable when the power system operates steadily. Therefore the results of state estimation can be used as the comparing data resource. The precision is the second. Then, compare the measurement value of protection equipment with the reference value to detect hidden failure. When the system was un-dynamic states such as short circuit, the measurement data such as bus voltage and current would changes rapidly. In order to avoid the miscalculation caused by the sync data under dynamic states, the detection of hidden failure should be blocked. The process is illustrated in figure 1, and specific steps are as follows:

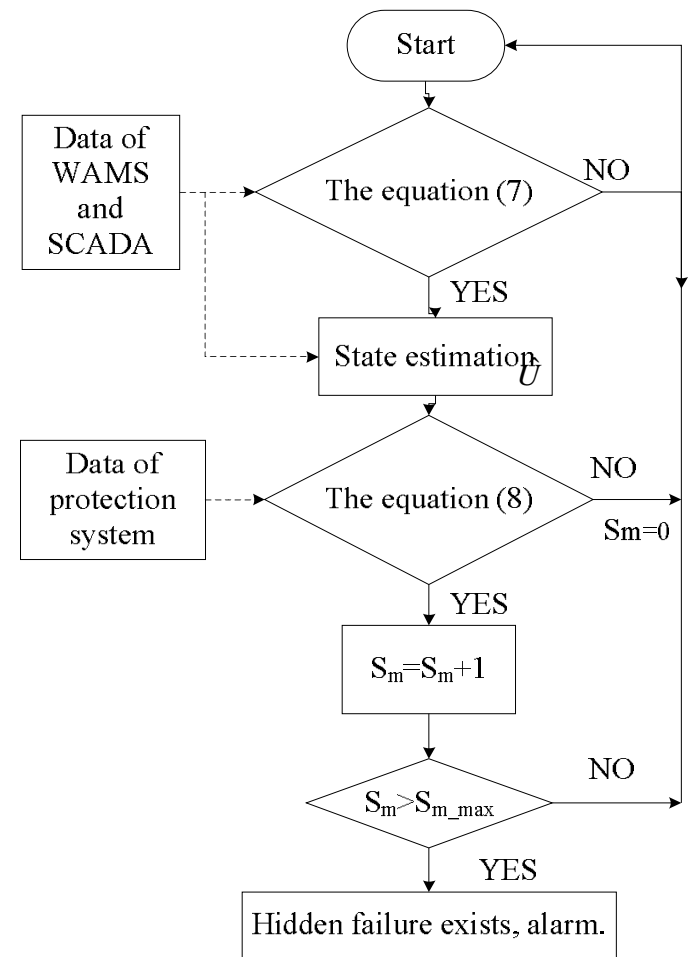

Fig1. The process of hidden failure detecting

Firstly, When the system was under dynamic states such as short circuit, the voltage amplitude of short circuit point will decrease. If the difference between the actual value and rated value of a bus voltage is over a certain number, the detection of hidden failure should be blocked because the system is operating under dynamic states.

The cycle time of $\mathrm{T}$ of detecting hidden failures is set. At time $\mathrm{k}$, the voltage amplitude of bus $\mathrm{j}$ updated from WAMS and SCADA system is $U_{j}(k)$. At time $\mathrm{k}+\mathrm{T}$, the voltage amplitude is $U_{j}(k+T)$. 
The data deviation of voltage amplitude is set to be $10 \%$. Then the deviation of voltage amplitude between two adjacent cycles should not pass $10 \%$. The equation is:

$$
\left|U_{j}(k+T)-U_{j}(k)\right| \leq 0.1 U_{N}
$$

If the above equation is satisfied, the system is operating steadily. The detection runs normally. If the above equation is not satisfied, the system is operating under dynamic states. The detection of hidden failure should be blocked until the voltage amplitude of this bus return to normal.

Secondly, if cycle time of $\mathrm{T}$ of detecting hidden failures is set, the protection equipment uploads $\mathrm{m}$ times in the time $\mathrm{T}$. And the uploading frequency is $\mathrm{f}=\mathrm{m} / \mathrm{T}$. The measurement data is processed and the hidden failure is checked on a $\mathrm{T}$ cycle. In the operation of practical power system, the sample data should not to be compared one by one. Therefore, in detecting cycle, this paper we proposed a method which obtains the mean square covariance between protection measurement data $U_{i}$ of the bus and reference values $\hat{U}$ obtained by state estimation in the sampling period. Set the threshold value $\varepsilon_{X}$. If the mean square covariance exceeds the threshold value $\varepsilon_{X}$, which satisfy the equation 7 , the data deviation is said to be too large.

$$
\frac{\sqrt{\frac{1}{m} \sum_{i=1}^{m}\left(U_{i}-\hat{U}\right)^{2}}}{|\hat{U}|}>\varepsilon_{X}
$$

Thirdly, if the mean square covariance exceeds the threshold value $\varepsilon_{X}$, the counter $S_{m}$ will plus one automatically. If the condition is not met, set the counter $S_{m}$ to zero. After n cycles, when the counter $S_{m}$ exceeds the maximum number $S_{m_{-} \max }$, hidden failure can be proved to exist in relay and the test system gives alarms.

\section{Analysis of Sync Data}

The detecting system of hidden failure includes two data sources, the data of power grid compiled from WAMS and SCADA system and the protection data compiled from protection information system. Because of the delay of data transmission, the data compiled by different resources may be out of sync.

This paper use voltage amplitude as sampled data to test the hidden failure under static state. When the power system operates steadily, the voltage amplitude changes pretty slowly ${ }^{[19-23]}$. The influence of sync can be ignored.

The difference of data accuracy between SCADA and WAMS system is huge. The solution of data compatibility in WAMS and SCADA detecting system can reduce the accuracy to second ${ }^{[19-20]}$. The power grid uses GPS system to improve the sync accuracy between SCADA/WAMS system and protection information ${ }^{[21-25]}$. The accuracy can be millisecond. And the requirement of data sync in this testing system is second. Therefore, the sync of data has no influence in this testing method.

Therefore, the sync of data caused by the steady of amplitude or the sync analysis of data resources has little influence in the method in this paper. 


\section{Analysis of Threshold of Hidden Failure}

At time $\mathrm{K}$, voltage amplitude is $\hat{U}$ after the state estimation of data measured by SCADA and WAMS. In the measuring period, voltage amplitude of phase A collected by protection information system is $U_{i}$. After transformation the equation 8 is:

$$
\sqrt{\frac{1}{m} \sum_{i=1}^{m}\left(U_{i}-\hat{U}\right)^{2}}>|\hat{U}| \varepsilon_{X}
$$

The alarming threshold value of detecting system for hidden failure is set to $\Delta U_{X}=|\hat{U}| \varepsilon_{X}$. The criterion of equation 8 changes to:

$$
\sqrt{\frac{1}{m} \sum_{i=1}^{m}\left(U_{i}-\hat{U}\right)^{2}}>\Delta U
$$

When lines keep stable operation, the actual data can be compiled by WAMS and SCADA system. And there are differences between the operation state measured by protection equipment and the operation state of practical power system, which means that it is different with the operation state collected by WAMS. The differences stem from all kinds of measurement and calculation errors which includes the measurement error of voltage transformer $\varepsilon_{\text {tran }}$, the state estimation errors $\varepsilon_{\text {state-est }}$, the calculation errors of relay protection equipment and so on. It can be expressed as:

$$
\varepsilon_{\Delta U}=\varepsilon_{\text {tran }}+\varepsilon_{\text {state-est }}+\varepsilon_{\text {relay }}
$$

Using the data uploaded by WAMS and SCADA could state-estimate the grid voltage and relinquish the bad data in order to obtain the voltage amplitude $\hat{U}$ of corresponding bus. It has high reliability and accuracy, and can be used as reference value. Then the relationship between the alarming thresholds $\Delta U$ of hidden failure in protection equipment, the measurement errors $\varepsilon_{\Delta U}$ and the voltage amplitude $\hat{U}$ is:

$$
\Delta U \geq \hat{U}\left(\varepsilon_{\text {tran }}+\varepsilon_{\text {state-est }}+\varepsilon_{\text {relay }}\right)
$$

Reliable coefficient is set to $K_{\text {rel }}$, and the threshold $\Delta U_{X}$ could be set to:

$$
\Delta U=K_{\text {rel }} \hat{U}\left(\varepsilon_{\text {tran }}+\varepsilon_{\text {state-est }}+\varepsilon_{\text {relay }}\right)
$$

The errors of measuring transformer and protective transformer influence the calculation of threshold. The protective transformer is put into protection equipment. At rated voltage, the error of magnitude 3 is $\pm 3 \%$, and the error of magnitude 6 is $\pm 6 \%$. In this paper, the protective transformer uses magnitude 3, which means that the error of measurement data uploaded to protection information system is about $\pm 3 \%$.

Under the steady state operation of power system, WAMS and SCADA upload the data of power grid, and both adopt measuring voltage transformer. At rated voltage, the errors of magnitude 0.1 , 
$0.2,0.5,1$ transformers are $\pm 0.1 \%, \pm 0.2 \%, \pm 0.5 \%, \pm 1 \%$. This paper adopt magnitude 0.5 transformer. Then the error of data updated by power system is about $\pm 0.5 \%$. The state estimation uses iteration which solves nonlinear equation to estimate the best estimated state of power grid. This method will produce calculation errors. And the processing of data in WAMS and SCADA system will produce other errors.

In IEEE 118-bus system, the original measurement in system is set to active and reactive power flows of all branches. The measurement error of measuring transformer, the calculating error of state estimation and other measurement error will affect the random error of state estimation. The measurement data of PMU and SCADA system is simulated by truth state value superposing on random errors. The random errors of SCADA system submit standard distribution which mean index is 0 and standard deviation is $0.02^{[16]}$. Bus 22 is configured with PWU. The measurement errors of amplitude submit standard distribution which mean index is 0 and standard deviation is $0.005^{[17]}$.

Under the steady state operation of power system, the deviation of data is very little (not over $10 \%)$. This deviation may affect the calculation of threshold. Therefore it should be analysis. And if the measurement of a bus in grid produces large deviation, according to weighted least squares, although the deviation is reduced by state estimation, the deviation of neighboring buses will increase. Therefore it is needed to be analyzed and the influence of threshold should be ensured. Assuming that the deviation in voltage amplitude of bus 40 is $0 \%,-10 \%$ and $-20 \%$ respectively, the state after estimation is compared with the state before estimation. The measurement error of power bus is shown in figure 2 .

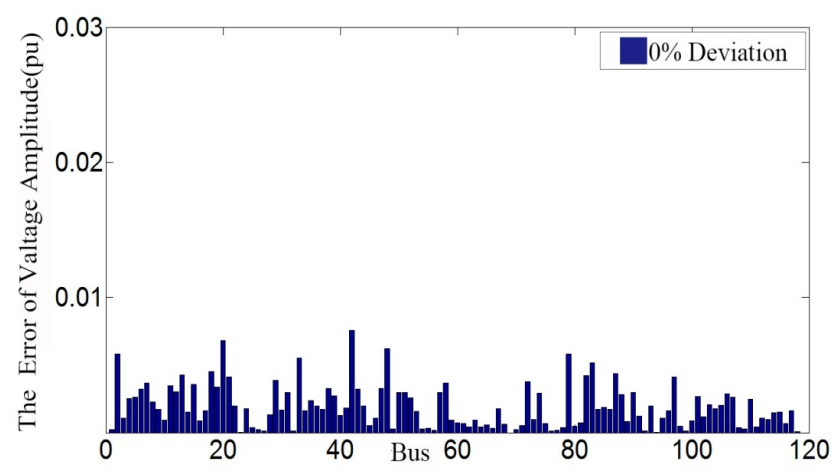

(a)

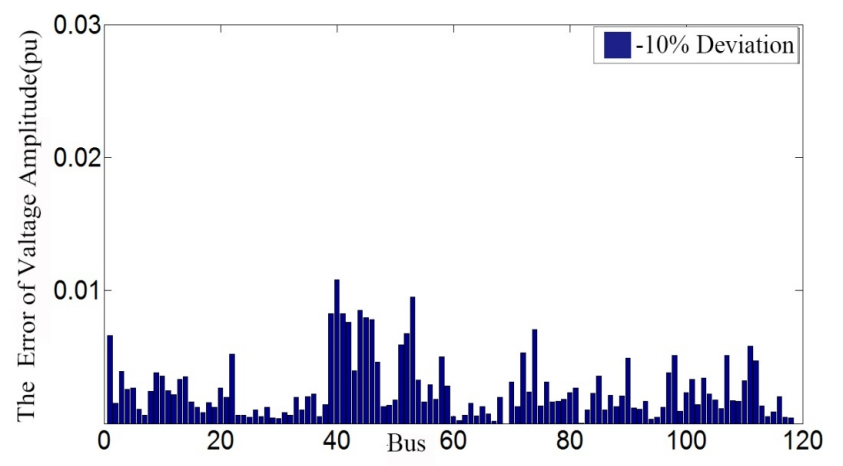

(b) 


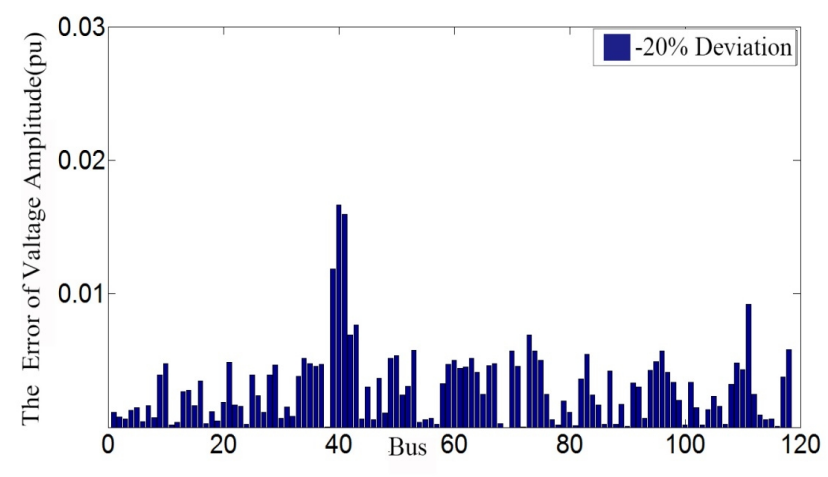

(c)

Fig2. The measurement error after state estimation compared with before

In figure 2, the bigger the deviation of a bus is, the bigger the calculation error is. However, state estimation could also reduce the deviation of measurement effectively and keep the steady of testing system in order to avoid misjudgment. In this paper, the measurement deviation of bus voltage amplitude is set to $-10 \%$. Therefore the deviation of state estimation is $1 \%$.

After calculating the discrete grid measurement data, the voltage and current of grid are uploaded to protection information system by protection equipment. Affected by software algorithm, frequency and the harmonic, there will be calculation error of voltage amplitude in this process. According to the calculation precision of microcomputer protection, the error is about $\pm 1.5 \%{ }^{[19]}$.

Reliable coefficient $K_{\text {rel }}$ is set to 1.2 . Based on the preceding analysis, $\varepsilon_{\text {tran }}=0.03, \varepsilon_{\text {state-est }}$ $=0.01, \varepsilon_{\text {relay }}=0.015$. According to equation 11, the threshold of hidden failure is:

$$
\Delta U_{X}=1.2^{*} \hat{U}^{\star}(0.03+0.01+0.015)=0.066^{*} \hat{U}
$$

\section{Detecting system for hidden failure}

A highly efficient information processing measurement is needed by safe operation of the power grid. Intelligent substation and its monitoring system is the inevitable trend of the development of modern power grid. SCADA, WAMS and protection information system are used as main means of monitoring grid and its equipment. And it can provide sufficient data sources to detect hidden failure. According to this paper, detecting system for hidden failure is shown in figure 3 . This system does not change the physical structure of original detecting system. It only need open the corresponding data query interface in SCADA, WAMS and protection information system. Detecting system for hidden failure isolates physically from original detecting network of power grid. Proprietary data network is used to communicate with SCADA, WAMS and protection information system and satisfy the call and measurement and the upper layer of signal data.

Detecting system for hidden failure proposed in this paper is mainly used for the discriminate under static condition. The function of the module and discriminate method are illustrated in detail in the earlier paragraphs. The detecting system of this system can achieve a online real-time operation. It will alarm whenever an exception of any component is detected and report the source of the error. 


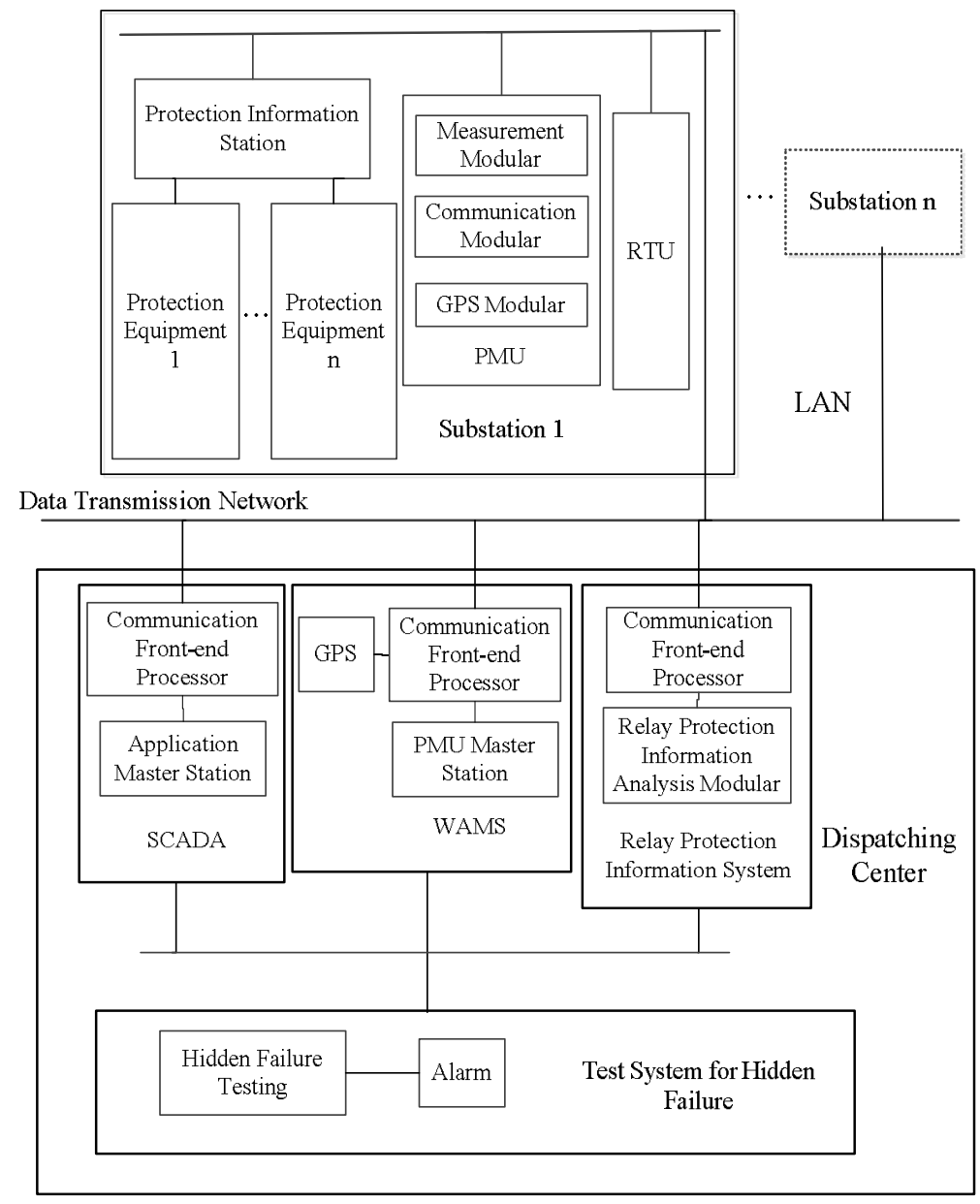

Fig. 3 Structure chart of detecting system for hidden failure

\section{Example}

The article selects IEEE 118-bus system shown in figure 4 as instance. Power flow calculation results calculated by program "matpower4.1" are used as real-time state operation of system.

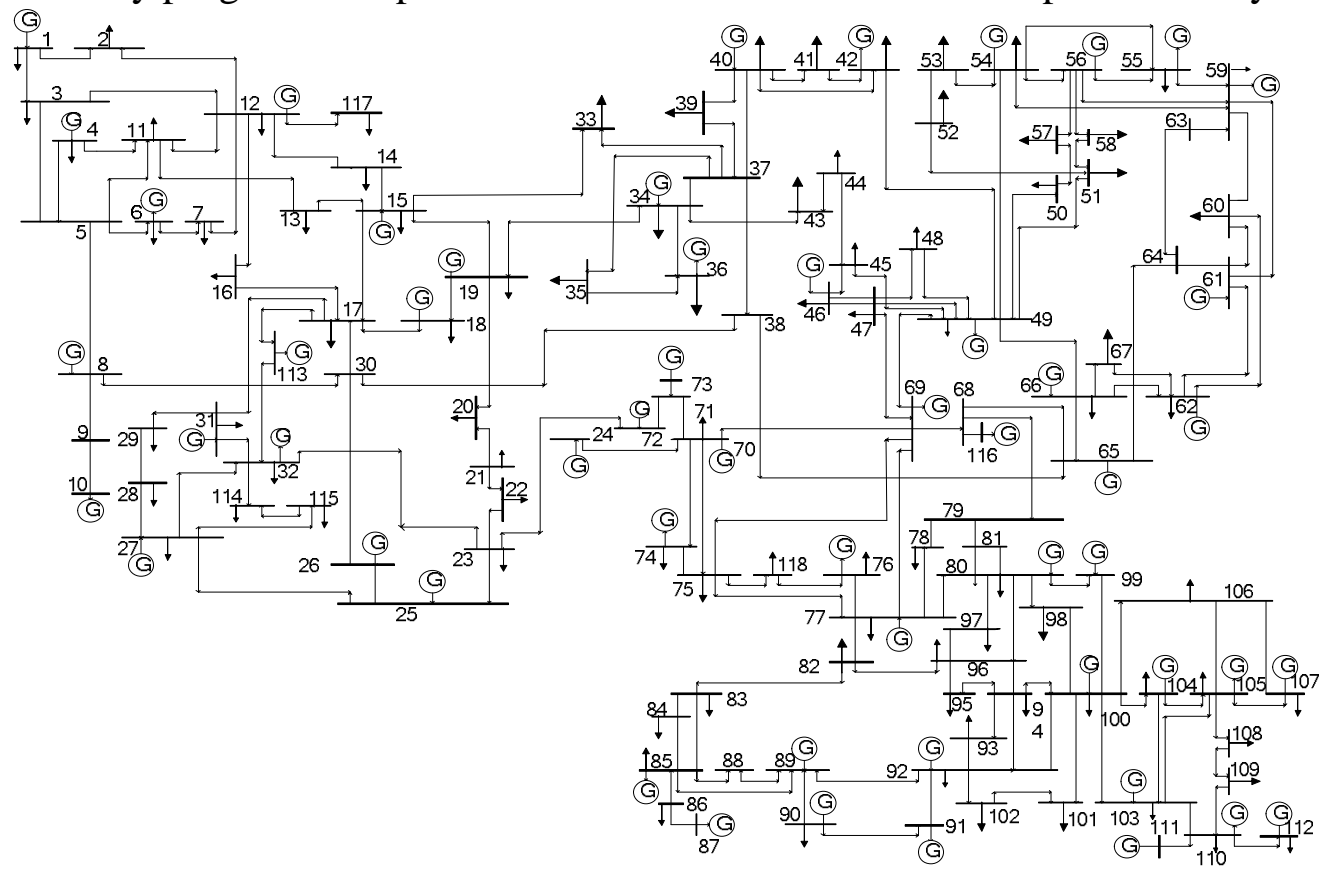

Fig. 4 IEEE 30-bus test system 
The state estimation and the measurement of protection information system are simulated by using truth flow value superposing on random errors. In state estimation, the measurement errors of SCADA and PMU are shown in section 1.4. The measurement error of the data in protection information system submits standard distribution which mean index is 0 and standard deviation is 0.02. The threshold is determined by equation 13. The analysis of detection for hidden failure is:

Firstly, if there is no hidden failure in the system, the magnitude $\left|U_{i}-\hat{U}\right|$ of the difference between the result of state estimation and the data of protection equipment at some time is shown in figure 5.



Fig. 5 Node amplitude differences without hidden failure

Secondly, assuming that bus 14 and 81 have hidden failures, the measurement error of voltage amplitude uploaded by protection information system is raised to $-10 \%$. Therefore the result after detecting is shown in figure 6.



Fig. 6 Node amplitude differences with hidden failure

Thirdly, if there has some deviation in WAMS and SCADA which leads to some deviation in protection measurement data, state estimation could reduce this deviation and ensure the accuracy of the detection. The deviation in the measurement of bus 20 is set to $-10 \%$. If there has no hidden failure, the result after detecting is shown in figure 7. If there have some hidden failures in bus 4 and 21 , the deviation of voltage amplitude uploaded by protection information system is $-10 \%$. The result after detecting is shown in figure 8. 


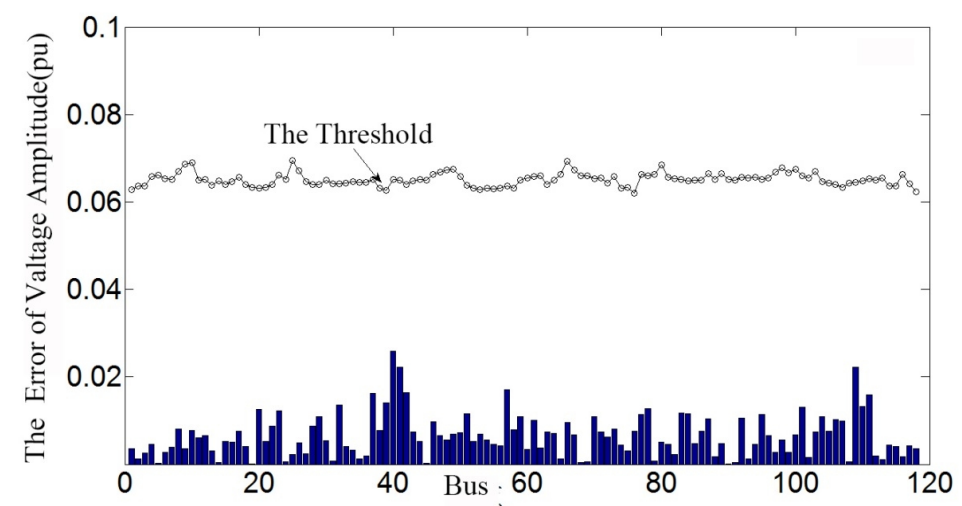

Fig. 7 Node amplitude differences without hidden failure with anomalous measured value

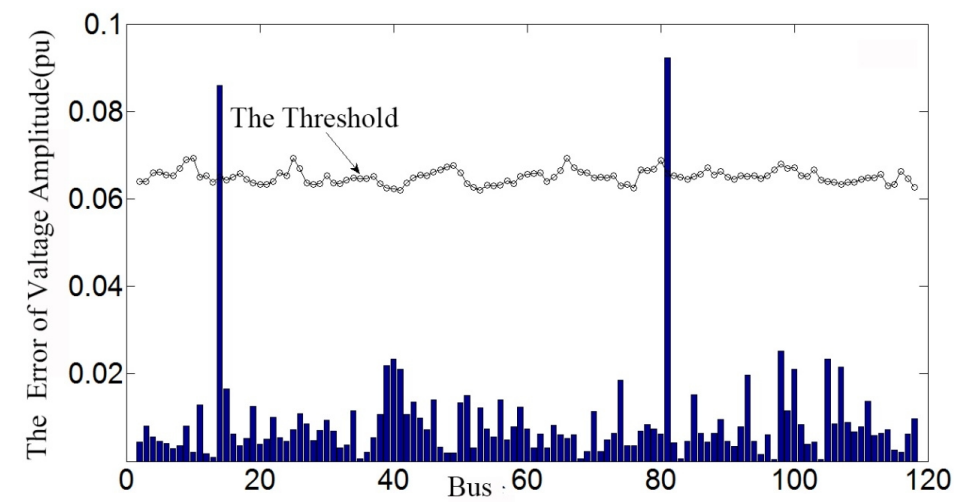

Fig. 8 Node amplitude differences with hidden failure in with anomalous measured value

In comparison between figure 5 and 6 , the measurement error of voltage amplitude caused by the hidden failure in protection equipment can be detected effectively by the method proposed in this paper. In comparison between figure 5 and 7, if there has some deviation in system data, this deviation can be reduced effectively. And the effect on reference value can be ignored, which would not cause the misjudgment of detecting hidden failure. In comparison between figure 7 and 8 , even if there has some deviation in measurement data, the detecting system can still detect hidden failure effectively.

\section{Conclusion}

This paper proposes a detecting system for hidden failure in protection equipment. This detecting system could detect the hidden failure in protection equipment under static condition. By hybrid state estimation, the calculated measurement data can be used as reference value of hidden failure and compared with the equipment data measured by protection information system. If the error exceeds the threshold set before, hidden failure can be proved to exist in relay under static condition. This system could detect the operation state of relay protection equipment and predict the possible hidden failure in relay. And when there has some deviation in bus measurement data, the system can still detect hidden failure effectively and has certain practical.

\section{Acknowledgements}

This research is supported in part by the new intelligent substation project, Research on Practicality of Protection, Control and Communication Technology, of State Grid Corporation of China. The project number is JBB17201200396. And the paper is also supported by National High-tech R\&D 
Program of China (863 Program), Research on the Processing, Storage and Application Technology of Mass Information from Power System. The project number is 2012AA050803.

\section{References}

[1] Elizondo D C, de La Ree J, Phadke A G, et al. Hidden failures in protection systems and their impact on wide-area disturbances[C]. Power Engineering Society Winter Meeting, 2001, IEEE, 2001,2: 710-714.

[2] Yin Yonghua, GuoJianbo, Zhao Jianjun,et al. Preliminary analysis of large scale blackout in interconnected North America power system grid on August 14 and lessons to be drawn[J].Power SystemTechnology,2003,27(10) : 8-11,16.

[3] Chen Weihua, Jiang Quanyuan, Cao Yijia.Risk assessment of power system cascading failure considering hidden failures of protective relays[J].Power System Technology,2006,30(13) : $14-19,25$.

[4] Phadke A G, Thorp J S.Expose hidden failures to prevent cascading outages [in power systems][J].Computer Applications in Power,IEEE,1996,9(3) : 20-23.

[5] Xiong Xiaofu, Cai Weixian, Zhou Jiaqi, Qin Jicheng, Wu Juan, Li Xuesong. Probabilistic Model for Transmission Lines Cascading Trips Caused by Hidden Failures in Relay Protection, Automation of Electric Power Systems,2008,32(14) : 6-10.

[6] Yi Jun, Zhou Xiao-xin. Cascading failure model of power grids considering frequency response characteristics and hidden failures[J].Automation of Electric Power Systems,2006,30(14) : 1-5.

[7] Yi Jun,Zhou Xiaoxin, Analysis on method of decreasing the risk of cascading failures in power systems based on cascading failure searching model. Power System Technology,2007,31(6) : 19-22.

[8] Zhou Zongfa, Ai Xin, Deng Huiqiong, et al. A method to analyze power system cascading failure based on fault tree and fuzzy reasoning[J].Power System Technology,2006,30(8) : 86-91.

[9] Xiong Xiaofu, Liu Xiaofang. Monitoring of static characteristics and diagnosis of hidden failures in protection system based on WAMS. Automation of Electric PowerSystems,2009,33(9) : 6-10.

[10] Ju Ping, Zheng Shiyu, Xu Qun, et al. Survey of wide area measurement[J].Electric Power Automation Equipment,2004,24(7) : 37-40. 
[11] Xu Shukai, Xie Xiaorong, Xin Yaozhong, Present application situation and development tendency synchronous phasor measurement technology based wide - area measurement system[J].Power System Technology,2005,29(2) : $44-49$.

[12] Li Hong, Li Weiguo. A new method of PMU placement for improving network observability and state estimation precision[J] . Journal of North China Electric Power University(Natural Science Edition),2011,38(1) : 31-34,106.

[13] Shi Li, Zhao Dong-cheng, Yang Ming-yu. Discussion on Hidden Failures in Relay Protection of Power Systems[J].Relay,2004, 32(5) : 66-69.

[14] Ren Jianwen, Zhou Ming, Li Gengyin. Research on comprehensive analysis and management system for power system fault information[J].Power System Technology,2002,26(4) : 38-41.

[15] Ni Yimin, Ding Jie, Zhao Jinrong,et al. PFS 3000 protective and fault information management system of power systems [J].Automation of Electric Power Systems,2003,27(17) : 86-88.

[16] Ding Junce, Cai Zexiang, Wang Keying. Mixed measurements state estimation based on WAMS[J].Proceedings of the CSEE,2006,26(2) : 58-63.

[17] Wang Keying, Mu Gang, Chen Xueyun. Precision improvement and PMU placement studies on state estimation of a hybrid measurement system with PMU[J].Proceedings of the CSEE,2001,21(8) : 29-33.

[18] Qin Xiaohui, Bi Tianshu, Yang Qixun. A new method for hybrid nonlinear state estimation with PMU[J].Automation of Electric Power Systems,2007,31(4) : 28-32.

[19] Li Dalu, Li Rui, Sun Yuanzhang. Data compatibility analysis of WAMS/SCADA hybrid measurements state estimation[J].Proceedings of the CSEE,2010,30(16) : 60-66.

[20] Liu Congshan, Liu Tianqi, Li Xingyuan, et al. Data Fusion Method of WAMS/SCADA Hybrid Measurements in Power System State Estimation. High Voltage Engineering,2013,2686.

[21] Zhao Hongga, Xue Yusheng, Gao Xiang, et al. Impacts of the difference between measurement transmission delays on state estimation and the countermeasures[J].Automation of Electric Power Systems,2004,28(21) : 12-16.

[22] Zhao Hongda. Power time synchronization system and its accuracy monitoring. Telecommunications for Electric Power Systems,2008,29(4) : 36-38.

[23] WANG Yu-dong, YOU Tian-qing. Study on time-synchronization networking of power system[J].Telecommunications for Electric Power System,2009,30(7) : 64-67. 
[24] Liu Wenxia, Luo Hong, Chen Jinshan. Communication delay analysis of WAMS considering network protection mechanisms[J].Automation of Electric Power Systems,2011,35(21) : 68-73.

[25] Zhu Lin, Wang Pengyuan, Shi Dongyuan. Status Monitoring Information Model and Configuration Description of Communication Network In Smart Substations[J].Automation of Electric Power Systems,2013,37 ( 11 ) : 88-92.

[26] Yu Yueping. Error causes of AC sampling measurement and its countermeasures[J].Electric Power Automation Equipment,2008,28 ( 11 ) : 118-121. 